\title{
Switching time between overt and covert speech: Generative attention
}

\author{
ROBERT J. WEBER, JAMES BLAGOWSKY, and REED MANKIN \\ Oklahoma State University, Stillwater, Oklahoma 74078
}

\begin{abstract}
Four experiments investigated a new phenomenon: the existence of a very large switching time effect that occurs from rapidly alternating between overt and covert (mouthed) speech. This is referred to as an intensity switching effect, and the time taken for each switch is, in itself, long enough for a spoken or mouthed character (letter or digit) to be produced. In Experiments 1 and 2, the intensity switching effect was shown to be different from the switching that occurs between categories of materials (letters and digits) because it is both much larger and much more resistant to practice effects. The intensity switching effect was also shown to be distinct from a memory load effect, since it holds even for perceptually available lists. In Experiments 3 and 4, the issue of a peripheral vs. a central origin of intensity switching was addressed. Evidence was found for a central origin. In addition, two models of response intensity representation were contrasted: a symbolic or digital model, with intensity altered by parameter substitution, and an analog model, with intensity represented by a moving pointer on an intensity continuum. The results supported the symbolic model. It is concluded that the intensity owitching effect is a measure of control processes at work in altering the intensity parameters of the vocal response system.
\end{abstract}

The usual discussions of switching time (see, e.g., Broadbent, 1971) involve receptive attention rapidly alternated from one channel or category to another. In contrast, the intensity switching phenomenon studied here involves generative or productive attention (Blagowsky, 1971; Weber \& Blagowsky, 1971), because there is a rapid switching or changing of response characteristics, while stimulus characteristics are minimized. ${ }^{1}$ The reader can readily demonstrate intensity switching of generative attention: First, record the time it takes to speak aloud the 26 letters of the alphabet as rapidly as possible. Next, do the same thing, but mouth or covertly speak the letters, so that they are either inaudible or just barely audible. Usually, fairly similar times will result for the aloud and silent modes (Weber \& Bach, 1969; Weber \& Castleman, 1970). Now, alternate between speaking the letters aloud and mouthing them.

One possible explanation for the intensity switching effect is that it results from alternating between different response categories, that is, moving from one list representation in memory to another in a cyclical manner. An example would be alternately saying letters and digits. If so, one would expect intensity switching effects to disappear rapidly with practice. Response category explanations are considered in Experiments 1 and 2. A second explanation involves memory load. In a

This research was supported in part by a grant to $R$. J. Weber from the Education Research Foundation, Oklahoma State University. The authors wish to acknowledge the assistance of Cathy Fiebig in the testing of subjects. Send reprint requests to Robert $J$. Weber, Psy chology Department, Oklahoma State University, Stillwater, Oklahoma 74078. J. Blagowsky is now at Southwestern Oklahoma State University, Weatherford, Oklahoma. R. Mankin is now with the Xerox Corporation, Rochester, New York. condition involving alternation of response intensity, the subject must remember not only where she/he is in the letter sequence but also whether the last response was aloud or mouthed in order to know the intensity of the next response. This type of explanation is considered in Experiment 2. A third type of explanation (Kelso, 1981) would place the switching time effect in the periphery of the motor system. Various dynamic loading or damping factors, such as muscle and jaw movements, laryngeal actions, and so forth, would require time to react as response intensity is changed. Peripheral vs. central explanations are addressed in Experiments 3 and 4. A fourth potential explanation for the intensity switching effect comes from the literature on motor control, and it centers on response planning time. Keele (1981), Kerr (1978), Posner (1980), and Sternberg, Monsell, Knoll, and Wright (1978) have all presented arguments and/or data to show that it takes time to plan a motor response or to execute that plan. Insofar as the present task involves changing a motor plan to alter output intensity, it would be expected to take time to do so. Thus, the switching time might be the outward manifestation of the time it takes certain control processes to alter response intensity plans. These control processes could work in at least two ways: symbolically, by setting intensity levels via passing a parameter to an intensity function, or analogically, by moving a pointer along an internal representation of an intensity continuum. These issues are considered in Experiments 3 and 4.

\section{EXPERIMENT 1}

The generality of the switching time effect was determined by comparing intensity switching time with cate- 
gory switching time. Intensity switching has been defined previously. Category switching involves alternating not between intensities but between categories of materials, such as numbers and letters. It is included in order to study the possibility that intensity switching is simply the result of alternating between response categories. In a departure from previous work on silent speech (Weber, \& Bach, 1969; Weber \& Castleman, 1970), the subjects in Experiment 1 mouthed letter or character names. This helped to keep scoring objective and was also shown in pilot work to produce results very comparable to silent speech conditions. In fact, the mouthing procedure often produces barely audible speech.

\section{Method}

Subjects. The subjects were 12 undergraduates who were paid $\$ 1.50$ each. They were tested individually for a period of approximately $1 \mathrm{~h}$.

Design and Procedure. There were two within-subjects factors, both at three levels: response intensity (speak, mouth, alternate between speaking/mouthing) by materials category (numbers, letters, alternating numbers/letters). In this and the subsequent experiments, the term "speak" refers to a normal speaking voice, such as is used in conversation. Conditions requiring alternation between numbers and letters are said to involve category switching; conditions requiring alternation between speaking and mouthing are said to involve intensity switching.

There were 10 internally randomized blocks of the nine conditions. For the number conditions, a sequence of 10 characters $(1,2,3,4,5,1,2,3,4,5)$ was generated, either by speaking, by mouthing, or by alternating between speaking and mouthing. For the letter conditions, a sequence of 10 characters ( $a, b, c, d$, e, a, b, c, d, e) was generated, either by speaking, mouthing, or alternately speaking and mouthing. For the number/letter conditions, a sequence of 10 characters $(1, a, 2, b, 3, c, 4, d, 5, e)$ was generated, either by speaking, mouthing, or alternating between speaking and mouthing. The production of a 10character sequence constituted a trial. The production time for completion of a 10-item sequence was the dependent variable.

For each trial, a cue card was placed in front of the subject with a sequence of 10 characters printed on it, as well as one of the cues "Speak," "Mouth," or "Speak/Mouth." When the subject finished studying the cue card, he/she turned it over, started a clock, and began generating the sequence. At the end of the sequence, the subject stopped the clock. Subjects were instructed to generate all sequences as quickly and as accurately as possible. Note that since the subject controlled the onset of the trial and generated a number of characters before stopping the clock, the procedure was somewhat different from the normal reaction time procedure. This same timing procedure was also used in the other experiments.

\section{Results and Discussion}

The principal results appear in Table 1 . The mean time per character (total time for the 10-item sequence divided by 10 ) is reported. There was a significant effect for materials category $[F(2,22)=10.69, p<.01]$, a significant effect for response intensity $[\mathrm{F}(2,22)=217.31$, $p<.01]$, and a significant Materials by Response Intensity interaction $[F(4,44)=40.19, p<.01]$. There were fairly comparable times for speaking and mouthing, approximately $.20 \mathrm{sec} / \mathrm{character}$. When the sequence involved both numbers and letters (category switching), the times increased to approximately $.26 \mathrm{sec} / \mathrm{character}$. The major findings involved those conditions requiring alternation between speaking and mouthing. For the alternate conditions, the times per character (.43 to $.49 \mathrm{sec})$ were approximately double that of strings that involved only speaking or only mouthing.

To better understand the process of alternating between response intensities, an index of switching time was computed, based on the following formula: Mean Time $/$ Switch $=[\mathrm{A}-(\mathrm{S}+\mathrm{M}) / 2] *(1 / 9)$, where $\mathrm{A}=$ alternate time, $S=$ speaking time, and $M=$ mouthing time, all for 10 -item sequences. The division by 2 gives the average time for generating the 10 -item sequence under the two response intensities, in this case, speaking or mouthing. The factor $1 / 9$ is used because there are only nine switches in a 10 -item sequence. As seen from Table 1, mean time per switch varies from .19 to $.32 \mathrm{sec}$, approximately the same time as required for the production of a character.

Although both intensity switching and category switching effects occurred, it is clear that alternating between intensities (.43 to $.49 \mathrm{sec} /$ character) takes much longer than alternating between number/letter categories (.25 to $.26 \mathrm{sec} / \mathrm{character})$. Evidently, intensity switching is much different from category switching. But could this simply be due to the relative unfamiliarity of the intensity switching task? Examination of practice effects indicated that although there were some practice effects for intensity alternation, there was no suggestion of

Table 1

Processing Time (in Seconds) per Character or per Switch to Generate Sequences as a Function of Response Mode and Materials

\begin{tabular}{|c|c|c|c|c|c|c|}
\hline \multirow[b]{3}{*}{ Response Mode } & \multicolumn{6}{|c|}{ Materials } \\
\hline & \multicolumn{2}{|c|}{ Numbers } & \multicolumn{2}{|c|}{ Letters } & \multicolumn{2}{|c|}{ Alternate Numbers/Letters } \\
\hline & Mean & $\mathrm{SD}$ & Mean & SD & Mean & SD \\
\hline Speak & .21 & .05 & .21 & .05 & $.26^{*}$ & .06 \\
\hline Mouth & .20 & .05 & .19 & .06 & $.25^{*}$ & .06 \\
\hline Alternate Speak/Mouth & $.47 \dagger$ & .10 & $.49 \dagger$ & .09 & $.43^{*} \dagger$ & .08 \\
\hline Mean Time per Switch & $.29 \dagger$ & & $.32 \dagger$ & & $.19 * \dagger$ & \\
\hline
\end{tabular}

Note-N $=12$. Mean time per switch is based on the formula given in the text.

* Category switching conditions. IIntensity switching conditions. 
reaching the performance efficiency achieved in the pure component tasks, speaking only or mouthing only. In contrast, category alternation times were rapidly approaching times for pure response modes. Of course, the present practice effects were based on a single session occurring on a single day. Examination of more extended practice effects was in order.

\section{EXPERIMENT 2}

This study addressed the following questions. Is the intensity switching time phenomenon a short-lived coding effect, as would be expected for category switch. ing? Or is it a more intrinsic process that is resistant to practice? That is, do intensity switching and category switching behave differently with respect to long-term practice? An additional question was concerned with whether the intensity switching effect is due to memory load caused by having to remember the sequence and keep track of the latest response mode; that is, can a simple memory load hypothesis explain the intensity switching effect?

\section{Method}

Intensity switching. A single, highly practiced subject was tested over a 10-day period, with four counterbalanced blocks of conditions per day. The conditions all involved the 10-letter sequence " $a, b, c, d, e, f, g, h, i, j$. ." There were four conditions: (1) Speak the sequence from memory, (2) mouth the sequence from memory, (3) alternate between speaking and mouthing from memory, and (4) alternate between speaking and mouthing with perceptual support. The perceptual support involved presenting the 10-letter sequence on a card to the subject, with the alternate letters enclosed in boxes. This indicated to the subject that the boxed letters were to be mouthed and the unboxed letters were to be spoken aloud.

Category switching. The same practiced subject also engaged in a category switching task for 5 additional days with four counterbalanced blocks per day. The 10 -item sequences were as in Experiment 1: letters (a, b, c, d, e, a, b, c, d, e), digits $(1,2,3$, $4,5,1,2,3,4,5)$, and category alternation $(1, a, 2, b, 3, c, 4, d$, 5 , e). These sequences were always spoken aloud.

\section{Results and Discussion}

Intensity switching. Figure 1 indicates the results for intensity switching conditions, with production time as a function of days. Clearly, there is no difference between times for sequence alternation when the sequence is entirely generated from memory or when there is a perceptual representation of the sequence in front of the subject. Thus, the intensity switching time effect does not seem to be dependent on memory load, at least with familiar sequences such as the present ones. Figure 1 also indicates that the intensity switching effect is very impervious to practice. The derived switching time function shows an initial time of approximately $.24 \mathrm{sec} /$ switch on Day 1 . There is a fairly regular decline in times through Day 6, at which time there is a dip. The sub. ject stated that he started using a rhythmic grouping

INTENSITY SWITCHING (a...j)

CATEGORY SWITCHING $(a . . . e, 1 . .5)$

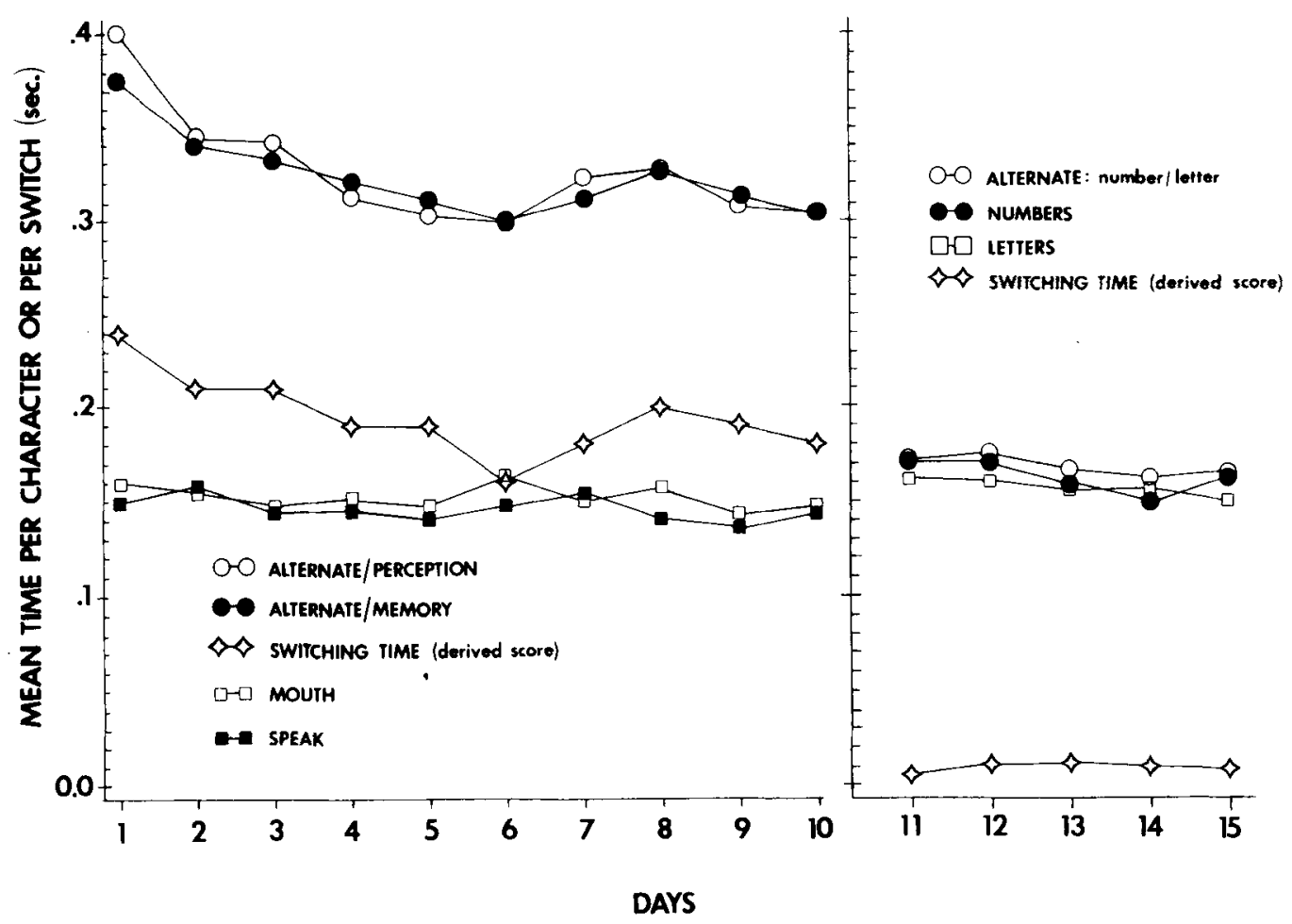

Figure 1. Mean processing time per character or per switch as a function of conditions and days of practice. 
strategy that he then abandoned on the next block. With a switching time of approximately $.19 \mathrm{sec} / \mathrm{letter}$ on Day 10 , there is no obvious indication that the intensity switching time effect will dissipate with practice.

Category switching. The right panel of Figure 1 depicts the category results for the same practiced subject. For a 10-character string, speaking aloud numbers or letters or alternating between numbers and letters takes very little difference in time; all are approximately $.16 \mathrm{sec} / \mathrm{character}$. As a result, the switching time that occurs in the alternating condition is virtually nil, as indicated by the bottom graph of Figure 1. (The switch. ing time formula discussed in Experiment 1 was also used here, with the exception that number and letter categories were used instead of intensity levels.) Clearly, category switching is a short-lived effect. This is not surprising, because the subject is probably just learning another string of characters that is no more arbitrary than either the letter names or digit names themselves or, for that matter, an alphabetic or numerical sequence.

\section{EXPERIMENT 3}

The argument for intensity switching would be strengthened if it were possible to go beyond a strictly dichotomous case of mouthing and speaking. In Experiment 3, intensity is manipulated over three levels, mouth, speak, and yell, where "mouth" has the same meaning as in Experiments 1 and 2, "speak" is, again a conversational level of intensity, and "yell" requires actual shouting. This manipulation should allow a distinction between intensity switching as a peripheral or as a central phenomenon; and, if it is a central phenomenon, the same manipulation should allow for a distinction between the symbolic and analogic models of central representation of intensity. The argument for the peripheral-central distinction is that increasing speech intensity will involve ever broader mechanical actions of the vocal apparatus (muscle, jaw, laryngeal movements, etc.). If so, then there should be a smaller switching time effect in changing from mouthing to speaking than from mouthing to yelling. The argument for distinguishing between control processes that operate symbolically or analogically is as follows: In a symbolic or discrete model, intensity is altered by passing different parameter values to an intensity function. In contrast, in an analogic model, intensity is selected by the position of a pointer on an internal intensity continuum. If the symbolic model is valid, it should take no longer to switch between mouth and yell than between mouth and speak. This is because it should not be any faster to substitute one symbolic parameter than another in an intensity function. In contrast, if the analogic model is valid, it should take longer to switch between mouth and yell than between mouth and speak (or speak and yell) because the pointer representing intensity on the intensity continuum would have to move a longer distance from mouth to yell than from mouth to speak (or speak to yell).

Note that the preceding predictions involve testing whether several conditions are the same or different in their generation times. Testing for differences involves standard statistical methods. However, testing for sameness-the typical case with scientific, as opposed to statistical, hypotheses (Polya, 1954)-requires an explicit statement of criteria: Processes shall be judged the same if their time scores "look about the same" (a subject matter decision) and they are not significantly different in a statistical sense. More will be made of this point in the Discussion.

\section{Method}

Subjects. The subjects were 10 male volunteers enrolled in introductory psychology classes; they received extra credit for their participation. Males were deliberately selected because a roughly uniform capacity for vocal intensity variation was desirable, and it was felt that males would be more willing than females to yell.

Procedure and Design. All subjects verbalized the string of 10 letters $(a . . j)$ in six different conditions: (1) mouth, a nonaudible mouthing of the letters, (2) speak, saying the letter string names at a normal conversational volume, (3) yell, shouting the letter string, (4) mouth/speak, alternating the intensity between successive letters (mouthing the first, speaking the second, etc.), (5) mouth/yell, alternating the intensity between successive letters (mouthing the first, shouting the second, etc.), and (6) speak/yell, alternating the intensity between successive letters (speaking the first, shouting the second, etc.). It should be noted that an alternation condition always had the same intensity applied to the same letters. Thus, for the mouth/speak condition, "a" was always mouthed, "b" was always spoken, "c" was always mouthed, and so forth. Each of the six ways of verbalizing the letter string was presented on a $4 \times 6$ in. cue card. The six cards, individually presented in random order, constituted one block. Each subject received 10 blocks.

The various conditions were modeled by the experimenter. A block of practice trials followed. Each subject pressed a switch with his left hand to start a millisecond clock when he began each letter string, and he pressed another switch with his right hand to stop the clock when a string was completed. Subjects were also instructed to respond as distinctly and as rapidly as possible. A cue card was presented and the subject started the clock when he was ready.

\section{Results}

Examination of Table 2 indicates that for the alone conditions, there were virtually identical times for mouth (M) and speak (S) conditions. The yell (Y) condition took significantly longer than the other conditions $[(M=S)<Y]$, with the Newman-Keuls multiple comparisons test requiring critical values of $\mathrm{C}$.diff $2=$ .028 and $\mathrm{C} . \operatorname{diff} 3=.034$ for $\mathrm{p}<.05$. This result suggests that the yell intensity level was sufficiently high to drive the speech output system into nonlinearity in the sense of increasing the time per item over that of the mouth and speak times.

The alternate conditions of Table 2 reveal a significant pairwise difference at the .05 level $[\mathrm{SY}<(\mathrm{MS}=\mathrm{MY})$, with critical values of $\mathrm{C}$.diff $2=.028$ and $\mathrm{C}$.diff3 $=.034$ ] . A comparison of the alone and alternate conditions of Table 2 indicates that it takes approximately twice as 
Table 2

Experiment 3: Processing Time (in Seconds)

\begin{tabular}{|c|c|c|c|c|c|c|c|c|}
\hline \multicolumn{3}{|c|}{ Alone } & \multicolumn{3}{|c|}{ Alternate } & \multirow{3}{*}{$\begin{array}{l}\text { Type of } \\
\text { Switch }\end{array}$} & & \\
\hline \multirow{2}{*}{$\begin{array}{l}\text { Response } \\
\text { Mode }\end{array}$} & \multicolumn{2}{|c|}{ Time per Letter } & \multirow{2}{*}{$\begin{array}{l}\text { Response } \\
\text { Mode }\end{array}$} & \multicolumn{2}{|c|}{ Time per Letter } & & \multicolumn{2}{|c|}{ Time per Switch* } \\
\hline & Mean & SD & & Mean & $\mathrm{SD}$ & & Mean & SD \\
\hline Mouth & .166 & .060 & Mouth/Speak & .384 & .098 & Mouth/Speak & .240 & .099 \\
\hline Speak & .169 & .053 & Mouth/Yell & .396 & .092 & Mouth/Yell & .236 & .084 \\
\hline Yell & .201 & .082 & Speak/Yell & .363 & .099 & Speak/Yell & .198 & .081 \\
\hline
\end{tabular}

Note $-N=12$. Based on switching time formula in text.

long to recite a sequence in alternating intensity as it does in uniform intensity $[\mathrm{F}(1,11)=255.86, \mathrm{p}<.0001]$. The important derived results for switching time are based on the equation from Experiment 1, except that in Experiment 3, a separate switching time was computed for each pair of intensities. The derived switching times are depicted at the right of Table 2. The mean times per switch indicate that speak/yell is significantly faster than mouth/yell and mouth/speak [SY < (MY = MS); Newman-Keuls $\quad$ C.diff2 $=.021$, C.diff3 $=.026 ; p<.05]$.

\section{Discussion}

Intensity itself has a minor effect on the rate of generating a sequence: For the alone conditions, yell took longer than mouth or speak. However, the effect of intensity on the alone conditions is very small in comparison to the effect of alternating intensities. When intensity is alternated, there is almost a doubling of generation time over the time required for a uniform inten. sity.

The most critical results for theory are the switching time values. Because the switching times for mouth/ speak and mouth/yell are virtually identical, it is argued that distance on the intensity continuum has no effect on switching time. Therefore, an analog model is contraindicated, and a symbolic parameter substitution model is supported. However, a different conclusion emerges when the speak/yell switching time is considered. Speak/yell involves adjacent intensities and produces a faster time than mouth/yell, which has nonadjacent intensity values. Hence, this comparison supports an analog model of intensity representation. The conclusion is splendidly ambiguous.

\section{EXPERIMENT 4}

Some of the results of Experiment 3 may have been due to a "chunking" effect, or rhythmic pattern of switching between intensity levels, that was made possible by always having, for a given alternation condition, the same letter at the same intensity level. Thus, subjects may have been able to plan several letters and intensities ahead when the same letter always had the same intensity in a given alternation condition. (This same problem was observed briefly in Experiment 2.) Experiment 4 was designed to break this possible pattern by balancing intensity levels for each letter. In addition, Experiment 3 suffered from an imprecise specification of speech intensity, and therefore there was probably substantial within- and between-subjects variability in the criterion of response intensity. That problem is addressed in Experiment 4 through the use of a sound-level meter to recurrently monitor speech intensity. Hence, the present experiment addresses the same issues as Experiment 3, but with some of the problems of control and procedure refined.

\section{Method}

Subjects. The subjects were 12 naive male volunteers from introductory psychology classes who received extra credit for participation.

Procedure. The same string of 10 letters was used $(\mathrm{a} . . \mathrm{j})$. The same six conditions were employed, with three additional conditions: speak/mouth, alternating between speak and mouth (to balance mouth/speak, which alternated between mouth and speak), yell/mouth (to balance mouth/yell), and yell/speak (to balance speak/yell). Thus, for each pair of alternating intensities, a given letter in the string $(a . \ldots j)$ received both levels of response intensity in order to break up consistent letter-intensity pairings.

In addition, a sound-level meter was used to make more objective the actual intensity levels of the speak and yell conditions. At 6 in. from the mouth, the sound level for speak was 76-80 dBA (reference level $=.0002 \mathrm{dyne} / \mathrm{cm}^{2}$ ). The sound level for the yell condition was $94-96 \mathrm{dBA}$. If sones are used as a measure of perceived loudness, then loudness increases from about 15 sones in the speak condition to about 40 sones in the yell condition (Licklider, 1951), approximately a threefold increase in loudness. Each subject monitored the sound-level meter throughout the experiment to maintain a proper level of response intensity. Other procedural matters were identical to those of Experiment 3.

\section{Results}

Table 3 indicates once more that for the alone conditions, mouth and speak are very similar, but yell takes longer $[(\mathrm{M}=\mathrm{S})<\mathrm{Y}$; Newman-Keuls C.diff2 $=.047$ and C.diff $3=.056 ; p<.05]$. The alternate conditions are averaged here for similar pairs, such as mouth/speak and speak/mouth, with the notation MS:SM. (There were no significant differences between such similar pairs.) The present results display a consistent pattern, with all alternate conditions statistically equal [NewmanKeuls C.diff $2=.047$ and $C$.diff $3=.056$ not reached at $\mathrm{p}<.05]$. 
Table 3

Experiment 4 : Processing Time (in Seconds)

\begin{tabular}{|c|c|c|c|c|c|c|c|c|}
\hline \multicolumn{3}{|c|}{ Alone } & \multicolumn{3}{|c|}{ Alternate } & \multirow{3}{*}{$\begin{array}{l}\text { Type of } \\
\text { Switch }\end{array}$} & & \\
\hline \multirow{2}{*}{$\begin{array}{c}\text { Response } \\
\text { Mode }\end{array}$} & \multicolumn{2}{|c|}{ Time per Letter } & \multirow{2}{*}{$\begin{array}{c}\text { Response } \\
\text { Mode }\end{array}$} & \multicolumn{2}{|c|}{ Time per Letter } & & \multicolumn{2}{|c|}{ Time per Switch* } \\
\hline & Mean & $\mathrm{SD}$ & & Mean & $\mathrm{SD}$ & & Mean & SD \\
\hline Mouth (M) & .233 & .095 & MS:SM & .429 & .114 & MS:SM & .226 & .116 \\
\hline Speak (S) & .221 & .088 & MY:YM & .453 & .105 & MY:YM & .203 & .125 \\
\hline Yell (Y) & .309 & .137 & SY:YS & .466 & .114 & SY:YS & .224 & .107 \\
\hline
\end{tabular}

Note $-N=12 . \quad{ }^{*}$ Based on switching time formula in the text, with the exception that the alternate conditions involving the same intensities (e.g., MS and SM) were first averaged for each subject.

A comparison of alone and averaged alternate conditions again reveals that it takes almost twice as much time to alternate intensities in the generation of a sequence as it does to produce the same sequence at a uniform intensity $[F(1,11)=54.16, p<.01]$.

The important switching time comparisons indicate a clear result: virtually identical times for each type of switch (MS:SM = MY:YM = SY:YS) and NewmanKeuls critical values of $\mathrm{C}$.diff2 $=.031$ and $\mathrm{C}$.diff3 $=$ .037 not reached at $p<.05$. This is evidence consistent with a symbolic model in which parameters are passed to an intensity function.

\section{Discussion}

Again, for the alone conditions, the speech output system is driven into nonlinearity when the intensity level becomes sufficiently loud; that is, the time per character increases for the yell condition. The alternate conditions require approximately twice as much time per item as the alone conditions.

Of greatest importance, however, is the finding of essentially equivalent switching times between all intensity levels. This is a result strongly contrary to a model in which intensity is represented on an analog scale, and it strongly supports a symbolic or parameter substitution model. In fact, the shortest time $(.203 \mathrm{sec} / \mathrm{switch})$ represents the longest intensity interval, mouth to yell.

\section{GENERAL DISCUSSION}

There is no doubt that the switching time effect is a very reliable and powerful phenomenon. It is not due to a lack of encoding practice, because it is very robust after 10 days of practice, whereas the category switching effect is not even present for a highly practiced subject. Nor is it due to nominal memory load, since it also occurs when there is perceptual support. The time involved in switching is relatively large; it is on the order of time required for the generation of another entire character. The results are also contrary to a model in which intensity is represented as an analog quantity, at least at the level of processing at which intensity selection is made. The results are consistent with a model in which intensity selection is made through symbolic parameter substitution. The same results that support a symbolic parameter passing model are contrary to any theory that would claim that the intensity switching effect is due to rapid activation and damping of peripheral components (muscles, jaw, laryngeal actions, etc.). Such a peripheral model would predict intensity effects in switching. Peripheral effects seem to be involved only in this sense: As the intensity increases to the level of a shout (yell), peripheral components are overdriven and the time per item increases. But because of the nature of the equation (from Experiment 1) for determining switching time, these intensity differences are taken into account exactly, and the underlying switching time is found to be invariant (Experiment 4) with respect to intensity. Thus, a central model is supported. The intensity switching effect would seem to be a measure of the time taken by control processes to change intensity parameters. Those control processes are themselves unaffected by the intensity values they handle.

At this point it is necessary to consider several issues involving alternative interpretations or potential problems of control. First, is the mouthing condition actually on the sound intensity continuum, or is it something qualitatively different from the speak and yell conditions? If mouthing is qualitatively different, then the issues addressed in Experiments 3 and 4 (but not Experiments 1 and 2) would not be adequately tested. Consider the following argument ${ }^{2}$ : It also takes a long time to switch between a low intensity of normal speech and an equally loud level of intense whispering. Clearly, there is some qualitative difference here, rather than an intensity difference, that is producing the switching time. That difference could be in central planning, but it could also be in the time it takes separate peripheral components in speaking softly and whispering equally loudly to be reset. Might not mouthing also be an example of a qualitatively different response mode? We argue in return that the mouthing condition was, in fact, on the same continuum as speaking and yelling: (1) When subjects performed the mouthing condition, it was not unusual for them to produce a very low level of audible speech. This would suggest a continuity between mouthing and speaking. (2) The times for the mouth-alone and the speak-alone conditions were virtually identical. If the mouthing condition was qualitatively different in an important way, then this similarity in times is merely 
coincidental and quite unexplained. Those studies (MacKay, 1981) that show that covert speech is faster than overt speech typically used unfamiliar sequences or low levels of practice. (3) In Experiment 4, the switching time between mouth and speak was essentially identical to that for speak/yell and for mouth/yell. Again, if mouthing is qualitatively, rather than quantitatively, different from speaking and yelling, then these similarities in times become yet another fortuitous and unexplained event. It would seem that the most parsimonious and consistent view is that mouthing is indeed on the same quantitative intensity continuum as speaking and yelling. The fact that there is also a switching time effect between a low level of normal speaking and an equally loud level of whispering should not be surprising. Switching time is likely to be a pervasive phenomenon, and it can be expected to occur between qualitatively different as well as quantitatively different responses.

Second, there is the potential problem of a speedaccuracy tradeoff (Wickelgren, 1977). Might not such a tradeoff be the basis of the switching time effect? The answer is clearly negative. A speed-accuracy tradeoff involves a negative correlation between response time and errors. In list processing tasks (Weber, Hochhaus, \& Brown, 1981), there is typically a positive correlation between time and errors. Under the conditions of the present study, those subjects with any degree of practice simply did not make errors in speaking overtly the alphabet or a subset of it. For the mouthing condition, it is of course difficult to assess objectively the error rate, but the subjective impression is also of errorless performance. Both the speak and mouth conditions were relatively fast. However, in the alternate condition, the response times were much longer and occasional "errors" did occur. They are not reported because they are difficult to classify: They ranged from occasional blocking to getting tongue tied. In those trials with a noticeable problem (fewer than $5 \%$ ), we would immediately rerun the trial, thus using a correction procedure. The net result is that the altemate conditions probably involved a slight underestimate of actual times. By the nature of the switching time equation, this would also mean that the switching times are also slightly underestimated. In short, insofar as errors are a factor, they contribute toward a conservative estimate of the size of the switching time effects.

Third, much of the reasoning in Experiments 3 and 4 centered on being able to go from a theoretical prediction of sameness to saying that empirical results for two or more conditions took the same time. Those schooled exclusively in statistical hypothesis testing will be very uncomfortable with such a pattern of reasoning. Nevertheless, it is a common pattern of reasoning in other sciences (Polya, 1954), and it of course involves plausible (scientific), rather than logical, inference. Indeed, it is a pattern of reasoning that is required whenever a prediction from theory is tested against data. There are no obvious statistical tests of sameness, but the various switching times "look the same," and they are not significantly different (Experiment 4).

Fourth, all of the experiments employed highly familiar materials, alphabetic lists or number lists. As a result, can any claim be made for the generality of the findings? The use of highly familiar materials was intentional. The aim was to minimize learning effects. Preliminary work with unfamiliar sequences, such as novel or scrambled sentences, has shown even larger switching time effects. Thus, the present results are probably conservative measures of the size of the intensity switching effect. One possible exception to this claim is the results for Experiment 2 , in which there was an absence of a memory load effect. It is entirely possible that the use of unfamiliar lists would be accompanied by a memory load effect.

Fifth, the results of Experiment 4 have been interpreted by some readers as support for a propositional theory of intensity representation (see Kosslyn \& Pomerantz, 1977, for a general discussion). We believe that it is more succinct and more accurate to say that vocal intensity is represented by passing an intensity parameter to an intensity function. After all, a function can be represented in a variety of formats, ranging from an equation to a list of ordered pairs to a geometric representation. Whether one wants to refer to any or all of these representations as propositional may be more a matter of definitional convenience rather than psychological reality. The further characterization of intensity parameters and intensity functions awaits study.

Sixth, little has been said about attention, even though the phrase "generative attention" was used earlier. The best immediate evidence for attentional involvement is subjective: Rapidly alternating between intensities requires great effort in a cognitive sense. Clearly, there is a need for greater specification of the relation between switching time and generative attention.

In conclusion, it is evident that the switching time paradigm has considerable potential for the study of those control processes that must be at work in the selection of response intensity and perhaps other response characteristics.

\section{REFERENCES}

Blagowsky, J. D. Metered memory search with concurrent response switching. Unpublished doctoral dissertation, Oklahoma State University, 1971.

Brondbent, D. E. Decision and stress. London: Academic Press, 1971.

KaPLAN, H. M. Anatomy and physiology of speech. New York: McGraw-Hill, 1971.

KEELE, S. W. Behavioral analysis of movement. In V. Brooks (Ed.), Handbook of physiology: Section 1: The nervous system (Vol 2). Motor control (Pt. 2). Baltimore, Md: American Physiological Society, 1981.

KeLso, J. Contrasting perspectives on order and regulation in movement. In J. Long \& A. D. Baddeley (Eds.), Attention and performance IX. Hillsdale, N.J: Erlbaum, 1981.

KERR, B. Task factors that influence selection and preparation for voluntary movements. In G. E. Stelmach (Ed.), Information 
processing in motor control and learning. New York: Academic Press, 1978.

Kosslyn, S. M., \& Pomerantz, J. R. Imagery, propositions, and the form of internal representations. Cognitive Psychology, $1977,9,52-76$.

LiCKLIDER, J. C. Basic correlates of the auditory stimulus. In S. S. Stevens (Ed.), Handbook of experimental psychology. New York: Wiley, 1951.

MACKAY, D. G. The problem of rehearsal or mental practice. Journal of Motor Behavior, 1981, 13, 274-285.

PoLyA, G. Patterns of plausible inference (Vol. 2). Princeton: Princeton University Press, 1954.

Posne R, M. I. Orienting of attention. Quarterly Journal of Experimental Psychology, 1980, 32, 3-25.

Ste rnberg, S., Monsell, S., KNolt, R. I., \& Wright, C. E. The latency and duration of rapid movement sequences: Comparisons of speech and typewriting. In G. E. Stelmach (Ed.), Information processing in motor control and learning. New York: Academic Press, 1978.

WEBER, R. J., \& BACH, M. Visual and speech imagery. British Journal of Psychology, 1969, 60, 199-202.
WEBER, R. J., \& Blagowsky, J. Metered memory search and concurrent chanting. Journal of Experimental Psychology, 1971, 89, 162-170.

Weber, R. J., \& Castleman, J. The time it takes to imagine. Perception \& Psychophysics, 1970, 8, 165-168.

Weber, R. J., Hochraus, L., \& Brown, W. D. Equivalence of perceptual and imaginal representation: Developmental changes. In J. Long \& A. D. Baddeley (Eds.), Attention and performance IX. Hillsdale, N.J: Erlbaum, 1981.

WiCkelgren, W. A. Speed-accuracy tradeoff and information processing dynamics. Acta Psychologica, 1977, 41, 67-85.

\section{NOTES}

1. The intensity switching effect reported here was discovered by Robert J. Weber and James Blagowsky around 1970 (Blagowsky, 1971). It is only recently that we have begun to understand the significance of the effect for motor control theory.

2. We are indebted to David Rosenbaum for this alternative interpretation.

(Received for publication February 22, 1982;

revision accepted July 27,1982 .) 